\title{
Postoperative Vaginal Brachytherapy of a Patient with Endometrial Cancer: Case Presentation
}

\author{
(1) Hakan EREN, (1) Yusuf Ziya HAZERAL, (1) Senem ALANYALI, (1) Zeynep ÖZSARAN \\ Department of Radiation Oncology, Ege University, Faculty of Medicine, İzmir-Turkey
}

\begin{abstract}
SUMMARY
Case: A 39-year-old woman presented with an abnormal postmenopausal vaginal bleeding, and probe curettage was performed for diagnostic purposes. She was diagnosed with endometrial cancer. She underwent total abdominal hysterectomy, bilateral salpingo-oophorectomy, and pelvic lymph node dissection (TAH+BSO+PPLND). Pathology specimen revealed endometrioid adenocarcinoma sized $2 \mathrm{~cm}$ with histologic grade 2 . Tumor had half inner myometrial invasion. Lymphovasculary invasion and lymph node metastases $(0 / 17)$ were not reported. She was referred to the radiation oncology department for intracavitary brachytherapy.

Keywords: Endometrial cancer; quality control; three dimensional (3D) brachytherapy treatment planning.

Copyright $\odot$ 2019, Turkish Society for Radiation Oncology
\end{abstract}

\section{Introduction}

Treatment Steps of Postoperative Three-Dimensional (3D) Brachytherapy

1. Gynecological examination: Pre-treatment gynecological assessment is necessary to rule out the early recurrences, to ensure postoperative healing of the vaginal stump, and to choose the optimal type and the size of the applicator. For optimal healing, at least a 4 -week wait between operation and brachytherapy is needed.[1]

2. Applicator selection: The ABS (American Brachytherapy Society) recommends that applicator selection should be based on patient anatomy, target volume geometry, and physician judgment.[2,3] However, if cylinder size is inappropriate for the patient, air gaps between the surface and applicator may occur, and they may cause low-dose areas. On the other hand, no study regarding the difference patterns of recurrences between ovoid and cylinder applicators has been published to date. For the patient mentioned above, no healing prob- lem was seen in gynecological examination, and a 3.5$\mathrm{cm}$ sized multichannel applicator was chosen.

3. Application: Patient should be informed about the procedure, and consent form should be taken in advance. Enema is recommended a night before the application. Placing the applicators horizontally also helps to reduce rectum and bladder doses. [4,5] In our daily clinical practice, we empty the bladder with the Foley catheter and filled with $150 \mathrm{cc}$ isotonic saline solution. Placing seeds or marking the vaginal stump with the radiopaque material would help to ensure the close contact of the applicators and the vaginal stump. In our department, we do not usually place seed but give extra attention to the applicator position during insertion. Multichannel cylinders provide advantage in regard to dosimetric goals such as improving target coverage and decreasing dose to the rectum and bladder.[2] We routinely use multichannel applicators in our department.

4. Imaging: To define target volumes, $3 \mathrm{D}$ imaging is being used. After the insertion of the applicator, we 
transfer our case to the CT (computed tomography) simulator with a dedicated bed system that prioritizes stabilization of the patient. Images acquired at slice thickness of $3 \mathrm{~mm}$ were loaded to the planning system.

5. Contouring: We follow European Society for Radiotherapy \& Oncology (ESTRO) recommendations for target volume contouring.[3] According to ESTRO, protocol clinical target volume (CTV) is defined as upper third of the vagina that is $3-5 \mathrm{~cm}$ long and $0.5 \mathrm{~cm}$ deep. Similarly, ABS also recommends to treat $3-5 \mathrm{~cm}$ of the vagina and to prescribe the dose to the surface or $0.5 \mathrm{~cm}$ depth of the vagina.[2] In our case, according to the risk assessment, CTV was defined as $4 \mathrm{~cm}$ of the vagina, and dose was prescribed to the $0.5 \mathrm{~cm}$ depth of vagina. Dose per fraction is 7 Gy. Organs at risk include bladder, rectum, sigmoid, perineal skin, urethra, and small intestine. To decrease the side effects, the dose of these organs should be kept below the recommended doses. The ABS guideline recommends to report the dose of $2 \mathrm{cc}$ (D2cc)s of the OAR. Therefore, to keep D2ccs for rectum, sigmoid, and bladder less than 70-75 Gy, $75 \mathrm{~Gy}$, and $90 \mathrm{~Gy}$, respectively, are appropriate.

6. Applicator definition: The catheters of multichannel applicator with a $3.5-\mathrm{cm}$ diameter were manually identified in the CT of an operated patient with endometrial cancer who had a 3-mm slice thickness in HDR brachytherapy treatment planning (Fig. 1).

7. Source loading: The 3D imaging provides visualization of the catheter location because of anatomy, calculation of the dose in every part of the patient, the location and amplitude of the brachytherapy nature, and the calculation of dosimetric indices. These al- low the physician to personalize the dose distribution as per each patient's anatomy. According to CTV, an active source position of $4.5 \mathrm{~cm}$ was determined with $0.5-\mathrm{cm}$ step size for all nine catheters (Fig. 2). The dose normalization point of the patient was manually determined to a depth of $0.5 \mathrm{~cm}$ from the surface of the multichannel applicator.

8. Dose prescription: The current source (source activity controlled by certificates and measurements) was selected, and the patient's dose was defined as the normalization point and the $100 \%$ isodose line with 7 Gy and three fractions.

9. Dose calculation: The dose calculation protocol of AAPM TG-43 (AAPM: The American Association of Physicists in Medicine, TG-43: Task Group 43 ) is accepted as the standard protocol, and is used in all commercial brachytherapy treatment planning systems.[6,7] Nucletron Oncentra Brachy v4.5.1 treatment planning system, which can perform the $3 \mathrm{D}$ brachytherapy planning in our clinic, uses AAPM TG 43 in dose calculation.

10. Dose volume histogram (DVH): The new generation of $3 \mathrm{D}$ planning systems can generate DVHs. Hence, the dose distribution of any contoured target volume and organs at risk can be defined by its DVH. The calculated dose information of the patient optimized by the "manual Dwell weight/times" method [8], and the DVH is shown in Figure 3.

11. Quality control: Generally, multichannel cylinder brachytherapy applicator is used as an applicator in the gynecological brachytherapy applications. After each use of these applicators, it is necessary to sterilize and preserve their sterilization until application. Place-

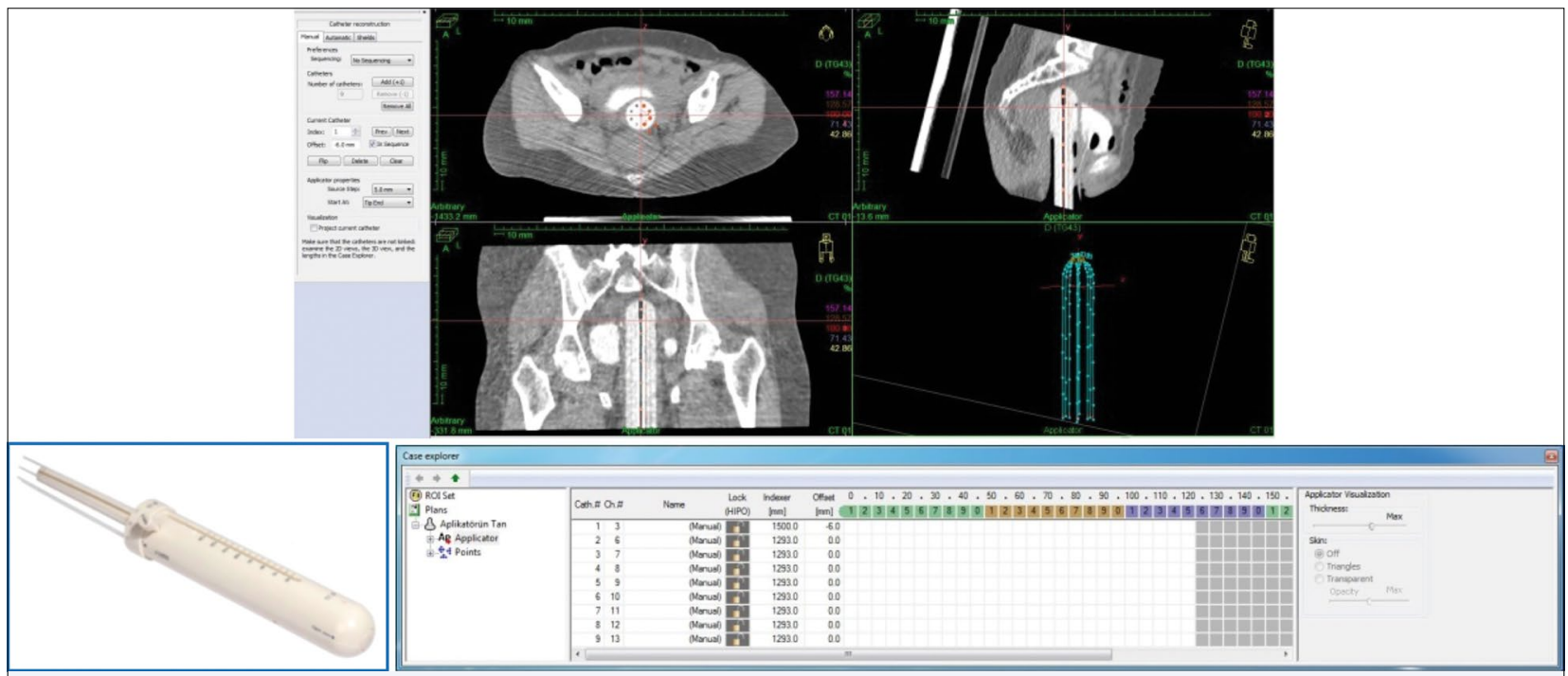

Fig. 1. Information of the Patient's multichannel catheters. 


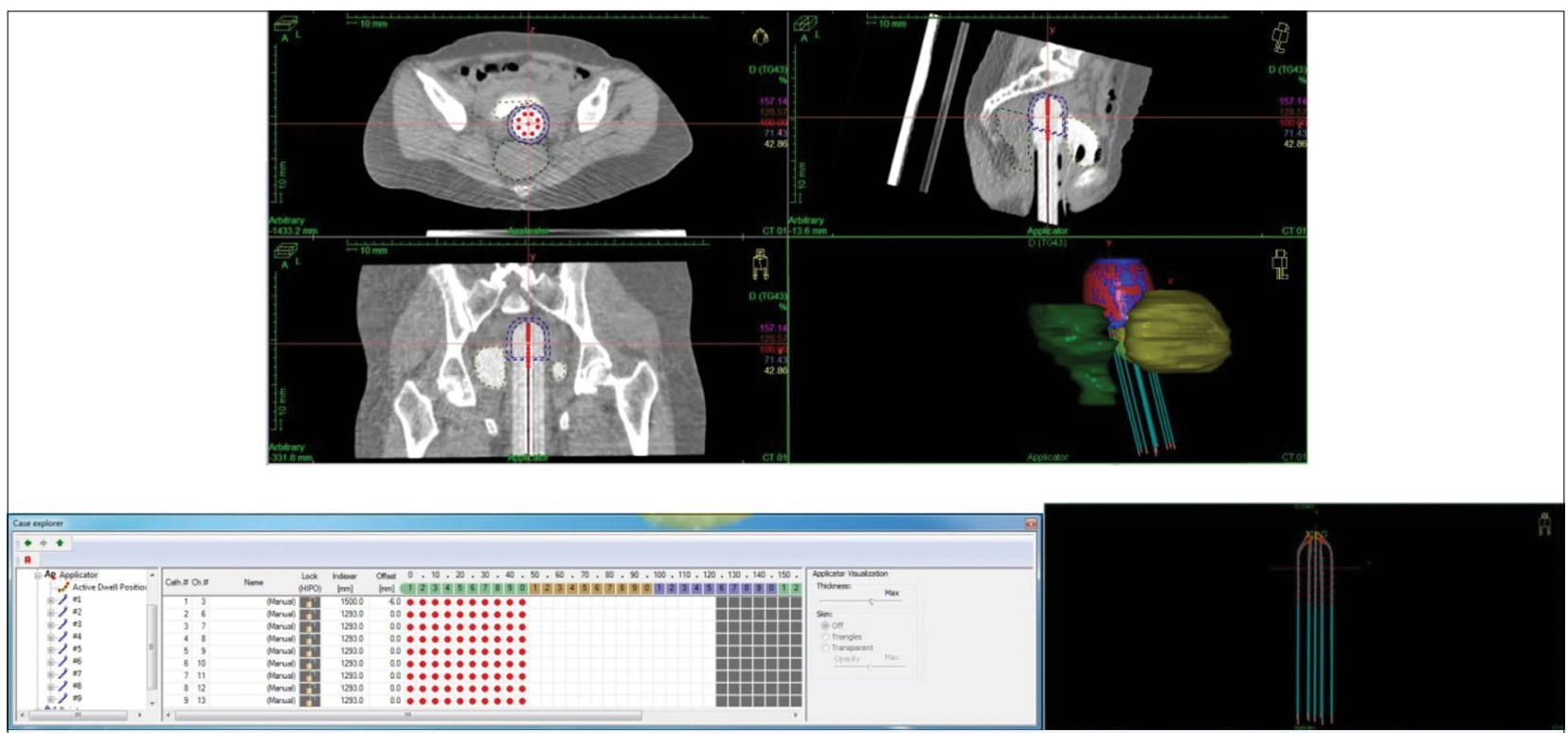

Fig. 2. Information of the Patient's source loading.

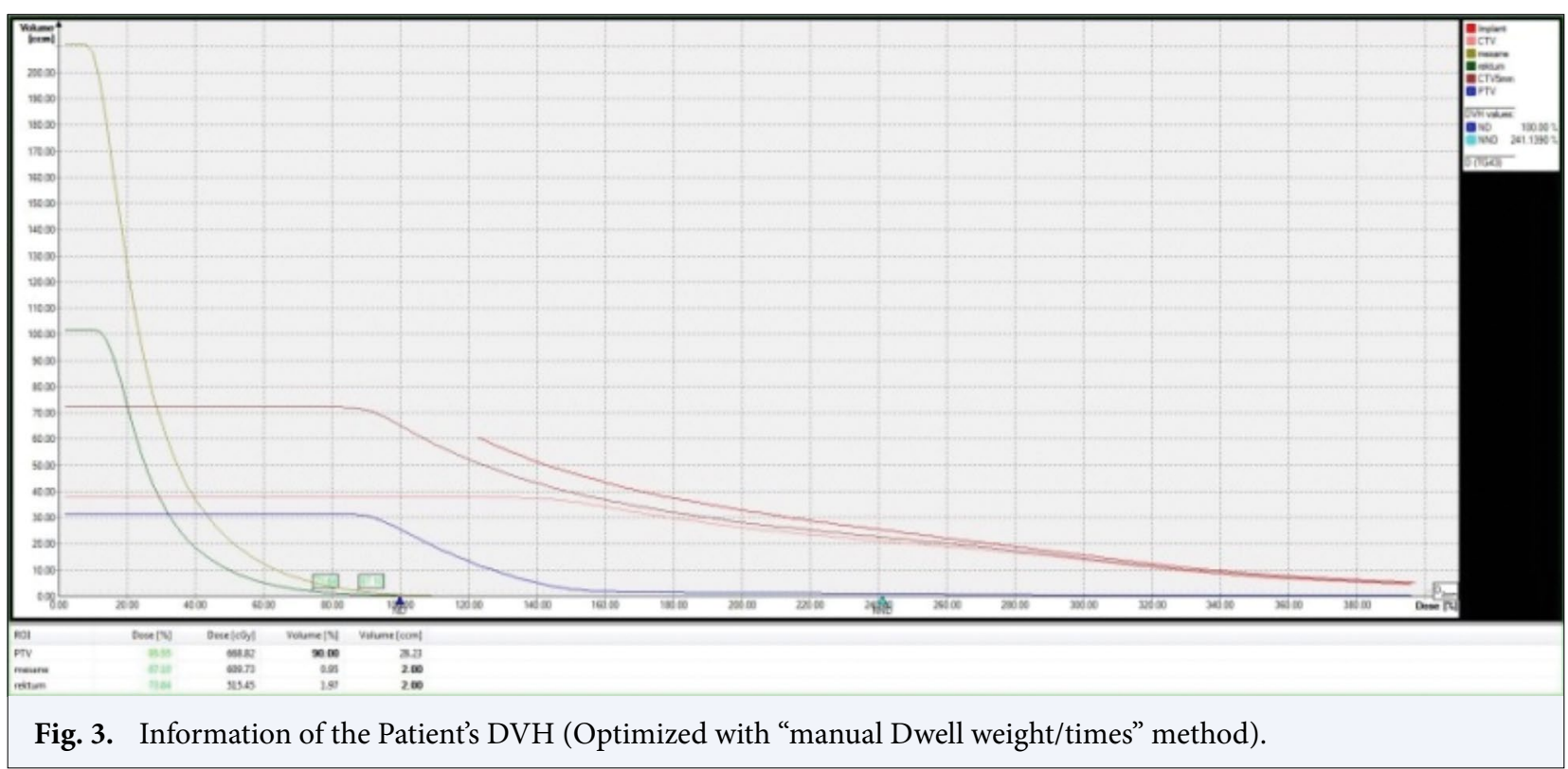

ment of the applicator to the patient is performed in a suitable application room in the clinic or operating room. The patient must then be transferred to the CT device for imaging. Care should be taken to ensure the stability of the applicator in the patient transport operations between the application room-CT and the CTtreatment room. Some developed patient transfer and treatment table systems (DIACOR Zephyr HDR) are available (Fig. 4).

After the scan, the patient's data are sent to the planning system in the DICOM format. After the contour- ing, the treatment information indicating the treatment dose and fraction as well as the applicator diameter and filled applicator catheters should be reported in writing before starting the planning process. Some literature state that non-written notifications can cause accidents by causing confusion and some accident reports that are formed by this lack of application. While the treatment plan is done, applicators should be careful about the orientation markers. There are several stages during brachytherapy treatment planning, and once these have been appropriately done, the patient plan- 


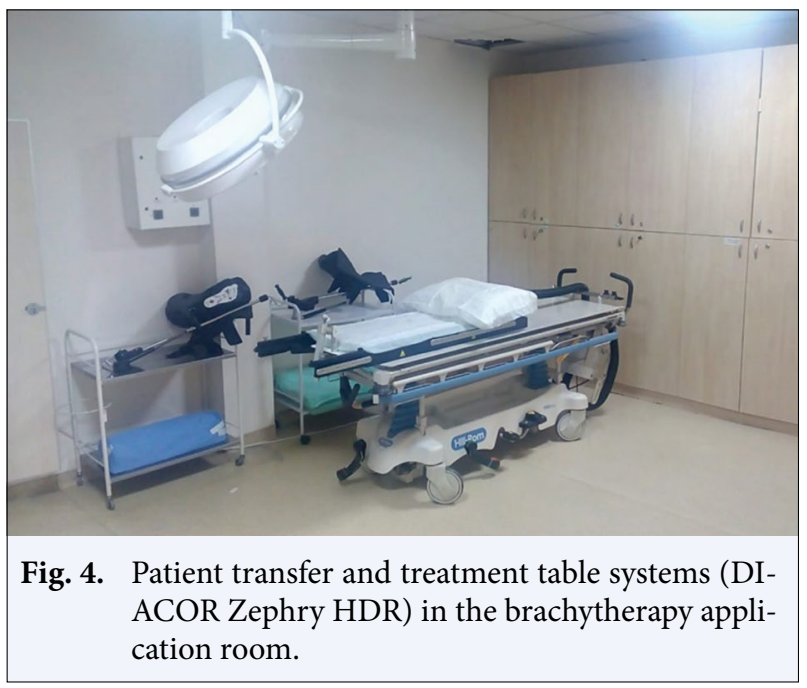

ning information data are taken through a printer for reporting and control. All stages of the treatment plan approved here are checked with print data. This set of data is a summary of the accepted treatment plan and shows all stages. Print data contain the following parts: Patient Information, Treatment Unit, Calibration Data, Treatment Data, Dose Normalization, Optimization and Prescription, Source Positions, Catheter (channel) Times, Total Treatment Time, Source Coordinates and Times, Applicator Points. Our patient plan sent to the treatment device is uploaded to the system and checked with print data. Here, the patient information and all the catheters of multichannel that are actively filled during the planning are checked. For the fraction, dose and total treatment time are checked by comparing the print data and the pre-treatment report. After the CT scan, the patient is transferred to the treatment device for treating, and then the cylinder orientation is checked after the applicator's catheters are connected to the treatment device. After print data and the treatment device have been checked, patient treatment is initiated, and the patient treatment is completed while the patient is followed up with camera systems.

\section{Conclusion}

Intracavitary brachytherapy is the preferred treatment modality in early-stage intermediate-risk group of patients. The 3D brachytherapy provides better delineation of the anatomic structures, dose evaluation with DVHs and optimization.
Informed consent: No.

Peer-review: Externally peer-reviewed.

Conflict of Interest: None declared.

Authorship contributions: Concept - H.E., Y.Z.H., S.A., Z.Ö.; Design - H.E., Y.Z.H., S.A., Z.Ö.; Supervision - H.E., Y.Z.H., S.A., Z.Ö.; Materials - H.E., Y.Z.H., S.A., Z.Ö.; Data collection \&/or processing - H.E., Y.Z.H., S.A., Z.Ö.; Analysis and/or interpretation - H.E., Y.Z.H., S.A., Z.Ö.; Literature search - H.E., Y.Z.H., S.A., Z.Ö.; Writing - H.E., Y.Z.H., S.A., Z.Ö.; Critical review - H.E., Y.Z.H., S.A., Z.Ö.

\section{References}

1. Monroe AT, Pikaart D, Peddada AV. Clinical outcomes of image guided radiation therapy (IGRT) with gold fiducial vaginal cuff markers for high-risk endometrial cancer. Acta Oncol 2013;52(5):1010-6.

2. Small W Jr, Beriwal S, Demanes DJ, Dusenbery KE, Eifel P, Erickson B, et al. American Brachytherapy Society consensus guidelines for adjuvant vaginal cuff brachytherapy after hysterectomy. Brachytherapy 2012;11(1):58-67.

3. Fokdal L, Tanderup K, Nielsen SK, Christensen HK, Røhl L, Pedersen EM, et al. Image and laparoscopic guided interstitial brachytherapy for locally advanced primary or recurrent gynaecological cancer using the adaptive GEC ESTRO target concept. Radiother Oncol 2011;100(3):473-9.

4. Richardson S, Palaniswaamy G, Grigsby PW. Dosimetric effects of air pockets around high-dose rate brachytherapy vaginal cylinders. Int J Radiat Oncol Biol Phys 2010;78(1):276-9.

5. Hung J, Shen S, De Los Santos JF, Kim RY. Imagebased 3D treatment planning for vaginal cylinder brachytherapy: dosimetric effects of bladder filling on organs at risk. Int J Radiat Oncol Biol Phys 2012;83(3):980-5.

6. Rivard MJ, Butler WM, DeWerd LA, Huq MS, Ibbott GS, Meigooni AS, et al; American Association of Physicists in Medicine. Supplement to the 2004 update of the AAPM Task Group No. 43 Report. Med Phys 2007;34(6):2187-205.

7. Beaulieu L, Carlsson Tedgren A, Carrier JF, Davis SD, Mourtada F, Rivard MJ, et al. Report of the Task Group 186 on model-based dose calculation methods in brachytherapy beyond the TG- 43 formalism: current status and recommendations for clinical implementation. Med Phys 2012;39(10):6208-36.

8. Tunçel N, Olacak N, Eren H. Brachytherapy Methods and Dosimetry Systems. Turkish Clinics Radiat Oncol-Special Topics 2017;3(1):25-36. 